\title{
Differential Roles of JNK in ConA/GalN and ConA-Induced Liver Injury in Mice
}

\author{
Hong-Min $\mathrm{Ni},{ }^{*}$ Xiaoyun Chen, ${ }^{*}$ Wen-Xing Ding, ${ }^{*}$ \\ Marcus Schuchmann, ${ }^{\dagger}$ and Xiao-Ming Yin* \\ From the Department of Pathology,* University of Pittsburgh \\ School of Medicine, Pittsburgh, Pennsylvania; 1st Department of \\ Medicine, ${ }^{\dagger}$ University of Mainz, Mainz, Germany
}

Tumor necrosis factor- $\alpha$-mediated liver injury can be induced by several different means; however, the signaling events and mechanisms of cell death are likely different. We investigated the mechanism of both apoptotic and necrotic hepatocyte cell death as well as the role of c-Jun $\mathrm{NH}_{2}$-terminal kinase (JNK) in the ConA and ConA/D-galactosamine (GalN) models of murine liver injury. ConA alone induced primarily necrotic cell death with no caspase activation, whereas ConA/GalN induced apoptosis in addition to necrotic cell death. The bi-modal death pattern in the ConA/GalN model was confirmed by the use of transgenic mice expressing a dominant-negative form of Fas-associated death domain in which the mice were resistant to apoptotic but not necrotic cell death. JNK1 and, more significantly, JNK2 participated in the induction of hepatocyte apoptosis in response to ConA/ GalN. Deletion of JNK led to the stabilization of FLIP $_{L}$, reduced caspase- 8 activation, decreased Bid cleavage, and inhibition of the mitochondrial apoptosis pathway. In contrast, JNK did not participate in necrotic death induced by ConA either alone or in combination with GalN. As such, JNK-deficient mice remained susceptible to necrotic liver injury in both model systems. Thus, ConA and ConA/GalN mouse models induce liver injury with different mechanisms of cell death, and JNK contributes to apoptotic but not necrotic cell death. These findings further elucidate the specific pathways involved in tumor necrosis factor$\alpha$-mediated liver injury. (Am J Pathol 2008, 173:962-972; DOI: 10.2353/ajpath.2008.080358)

Tumor necrosis factor (TNF) $\alpha$-induced liver injury is mediated by multiple signaling events, which are yet to be fully revealed. ${ }^{1-3}$ Liver injury can be attributed to hepatocyte death of apoptotic and necrotic nature. For the apoptotic mechanism, TNF $\alpha$ binds to its receptors and recruits the adapter molecule, TNF receptor-associated death domain, which engages Fas-associated death domain (FADD), which in turn binds to and activates the initiator caspase-8. FLICE-like inhibitory protein (FLIP) is structurally similar to caspase-8 with the N-terminal death effector domain, but the catalytic domain is either missing $\left(F L I P_{s}\right)$ or is inactive $\left(F L I P_{L}\right)$. It thus serves as an inhibitor of caspase- 8 activation by binding to FADD. While caspase- 8 can directly activate the downstream effector caspases such as caspase-3, to lead to cellular destruction, this activation is often blocked in the Type II cells, such as hepatocytes, by the X-linked inhibitor-of-apoptosis, which binds to caspase-3., ${ }^{4,5}$ Thus the cleavage of $\mathrm{Bid}$, a pro-death $\mathrm{BH} 3-$ only $\mathrm{Bcl}-2$ family protein, by caspase- 8 , is crucial to the death induction, as cleaved Bid (tBid) moves to the mitochondria to induce the release of cytochrome $c$, which activates caspase- 9 together with Apaf-1, and Smac, which overcomes the inhibitory effects of X-linked inhibitor-of-apoptosis. ${ }^{4-8}$ While the apoptosis pathway is relatively clear, very little is known about the necrotic mechanism of hepatocyte death in the context of TNF $\alpha$ toxicity, although in other types of cells this seems to be related to the receptor-interacting proteinmediated pathway and reactive oxygen species. ${ }^{9,10}$

The extent of hepatocyte apoptosis versus necrosis would certainly depend on the nature of the stimulation and the severity of the insults. In the experimental models of murine liver injury, TNF $\alpha$ could be directly administrated, or more frequently is induced in the animal. TNF $\alpha$ could be made by Kupffer's cells on stimulation by lipopolysaccharide (LPS), or by T lymphocytes on treatment with concanavalin A (ConA). In general, LPS-induced liver injury requires sensitization, such as by a liver-specific transcription inhibitor, D-galactosamine (GalN). This combination induces significant apoptosis of hepatocytes and irreversible liver damage with a high mortality.

Supported by an NIH grant to X.-M. Yin (R01CA 83817). W-X Ding was supported by a Liver Scholar Award from American Liver Foundation and Alpha-1 Foundation.

Accepted for publication June 26, 2008.

Address reprint requests to Xiao-Ming Yin, MD, PhD, Department of Pathology, University of Pittsburgh School of Medicine, Scaife Hall, $7^{\text {th }}$ Floor, Room S739, 3550 Terrace Street, Pittsburgh, PA 15261. E-mail: xmyin@pitt.edu. 
On the other hand, ConA-induced liver injury is more complicated. First, ConA alone is able to induce liver injury without sensitization. The injury is usually mild, reversible, and followed by liver regeneration. ${ }^{11-13}$ However, hepatocyte death is evident with the increased liver enzymes in the blood. The nature of the death could be necrotic, but not apoptotic, since caspase activity could not be identified at the time when blood enzyme level was peaked, ${ }^{13,14}$ although DNA breakdown could be detected. ${ }^{11,14,15}$ Second, sensitization with GalN clearly leads to a potent activation of apoptosis with significantly increased caspase activities. ${ }^{13}$ The liver damage becomes more severe and irreversible with a high mortality. Thus ConA/ GalN model seems to offer a better model to study the signaling events of the caspase-mediated apoptosis in this $T$ cell-mediated liver injury.

GalN blocks the transcriptional activity of nuclear factor $(\mathrm{NF})-\kappa \mathrm{B}$, which is concomitantly activated by $\mathrm{TNF} \alpha$, and suppresses the apoptotic effects of the latter. ${ }^{16}$ Gene products regulated by NF- $\kappa \mathrm{B}$ can suppress caspase and mitochondrial activation, reactive oxygen species accumulation and c-Jun $\mathrm{NH}_{2}$-terminal kinase (JNK) activation. ${ }^{16} \mathrm{JNK}$ has recently emerged as a key step regulated by NF- $\kappa$ B. Deletion of JNK1 or JNK2, or use of JNK inhibitors can suppress hepatocyte apoptosis in vitro and in LPS/GalN-induced liver injury in vivo. ${ }^{17-19}$ Intriguingly, it is reported that JNK1 could participate in the liver injury caused by the administration of ConA alone. ${ }^{15}$ However, the role of JNK in liver injury caused by ConA/GalN, where apoptosis is better characterized, has not been characterized.

Due to the unique presentation of liver injury in ConA versus ConA/GalN-treated mouse and the effect of GalN in blocking NF- $\kappa \mathrm{B}$, thereby promoting $\mathrm{JNK}$ activation, it is imperative to understand the role of JNK in the two different liver injury regimes that are related to the effects of ConA. Our present study provides definite evidence that JNK participates in only the apoptotic changes in the ConA/GalN model, but not the necrotic changes characterized by the ConA only model. This is consistent with the pro-death role of JNK being regulated by the NF- $\kappa \mathrm{B}$ activity.

\section{Materials and Methods}

\section{Animals}

JNK1-deficient mice (B6.129S1-Mapk8 tm1FN/J) and JNK2-de-

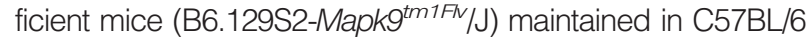
background were obtained from The Jackson Laboratory (Bar Harbor, ME). Transgenic mice expressing a dominant negative mutant of FADD in the liver had been described previously. ${ }^{20}$ All animals received humane care. Animal procedures were conducted according to the guideline of National Institute of Health with the protocols approved by the IACUC of the University of Pittsburgh.

\section{Reagents}

The following antibodies were used: anti-caspase 8 from Dr. Razq Hakem (University of Toronto, Canada); anticaspase-9, anti-caspase-3, anti-phosphorylated JNK, anti-phosphorylated c-Jun, and anti-c-Jun from Cell Signaling (Boston, MA); anti-B220, anti-CD3, anti-cytochrome c, anti-JNK (clone 666), and anti-Smac from BD Bioscience (San Diego, CA); anti-FLIP from Santa Cruz Biotech (Santa Cruz, CA); anti-Bid ${ }^{21}$ and anti- $\beta$-actin from Sigma (St. Louis, MO). ConA and GalN were also obtained from Sigma. They were prepared in 0.9\% endotoxin-free sterile saline (Sigma).

\section{Animal Experimentation}

Liver injury was induced as previously described. ${ }^{13}$ Briefly, mice about 20 to 30 grams in weight were intravenously given ConA ( $25 \mathrm{mg} / \mathrm{kg}$ ) with or without an intraperitoneal injection of GalN (700 mg/kg) given 30 minutes earlier. Mice were then sacrificed at the designated time point. Each time and treatment group contained 3 to 9 mice. For Western blot and caspase activity measurement, liver lysates from each mouse within the same treatment group were pooled to avoid any bias caused by conditions in individual mouse.

Bone marrow reconstitution was conducted as previously described. ${ }^{22}$ Recipient mice were lethally irradiated (1000 cGy) with a ${ }^{137} \mathrm{Cs}$ irradiator, fractionated in 600 and 400 cGy 4 hours apart. On the next day, they were intravenously reconstituted with $8 \times 10^{6}$ nucleated bone marrow cells from male donor mice. Recipient mice were challenged with ConA/GalN 12 weeks later. Number of mice used in each group is indicated in the figure legend.

\section{Biochemical Assays}

Serum level of alanine aminotransferase (ALT) was measured using the assay kit purchased from Biotron Diagnostics (Hemet, CA) according to the manufacturer's instruction. Serum TNF $\alpha$ and interferon $\gamma$ levels were determined using the Beadlyte mouse multicytokine detection system (Millipore, Billerica, MA), which is a Luminex-based multiplexed assay, per manufacturer's instruction. The experimental data were analyzed using a five-parametric-curve fitting.

Immunoblot assay and caspase activity measurement were conduced as previously described. ${ }^{7,23}$ JNK activity was measured using the in vitro JNK kinase assay with glutathione S-transferase-conjugated c-Jun as the substrate as described by the manufacturer (Cell Signaling).

\section{Flow Cytometry}

Single cell suspensions were prepared from mouse spleens after lysis of red blood cells. Cells were stained with fluorescein isothiocyanate-labeled anti-murine CD3 and PE-labeled antiB220 (BD Bioscience) and analyzed by a FACSCalibur flow cytometer. Data were analyzed with Win MDI software.

\section{Histology and Semiquantitative Assessment}

Livers were dissected, fixed in $10 \%$ neutral buffered formalin and paraffin embedded. Sections were cut at $5 \mu \mathrm{m}$ in thickness and stained with H\&E. Images were obtained 
A

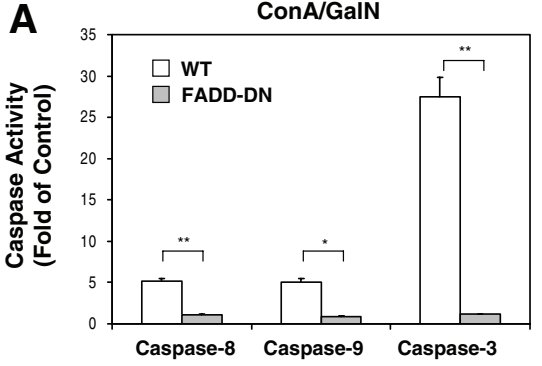

C

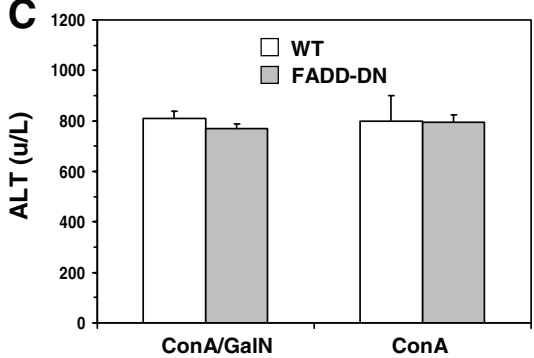

Figure 1. FADD-DN mice are protected from apoptotic, but not necrotic liver injury. A: WT and FADD-DN mice were treated with ConA/GalN (left panel) or ConA alone (right panel) and sacrificed at 8 hours later. The liver cytosol was analyzed for caspase-3, -8, and -9 activities using the corresponding substrates. Results (mean \pm SD) are expressed as the fold of changes over the non-treated control. Groups that had significantly different responses are indicated ( $t$-test; ${ }^{*} P<0.05 ;{ }^{* * *} P<0.01$ ). B: The liver cytosols of mice treated with ConA/GalN for different times were analyzed by immunoblot (cyto c, p15 cytochrome c, C-9: p49 pro-caspase-9 and p39 cleaved caspase-9, C-3: p20 and p19 cleaved caspase-3, and p37 GAPDH). C: Serum ALT levels (mean \pm SD) from ConA/GalN or ConA only-treated mice ( 6 or 8 hours, respectively). D: Liver sections of WT $(\mathrm{a}-\mathrm{c})$ and FADD-DN $(\mathrm{d}-\mathrm{f})$ mice treated with ConA/GalN $(b, e)$ or ConA alone $(c, f)$, or left untreated $(a, d)$ for 8 hours were H\&E stained and representative images were shown. Original magnification: $\times 100$.

using a light microscope (Nikon Eclipse E200, Melville, CA) equipped with a digital camera (SPOT, Diagnostic Instruments, Sterling Heights, MI). The degree of injury is assessed semiquantitatively by the following criteria and scored based on the area affected: a) increased eosinophilic staining of the hepatocytes and the accumulation of erythrocytes in the sinusoids (0: none; 1 : $<25 \%$; 2 : $25 \%$ to $<50 \%$; 3: $50 \%$ to $<75 \%$, and $4: 75 \%$ to $100 \%$ ), b) cellular vacuolization (0: none; 1 : $<25 \%$ and 2 : $>25 \%$ ), and c) cell Iysis (0: none; $1:<25 \%$ and 2: $>25 \%$ ). The final score of each sample is the summarization of the three parameters.

\section{Statistics}

Statistical analysis was performed using Student's t-test, where necessary.

\section{Results}

\section{FADD-DN Inhibits the Apoptotic, but Not the Necrotic Injury, in ConA/GalN and ConA Models}

To investigate the mechanisms of cell death in ConA/ GalN- and ConA-induced liver injury, we first examined
B

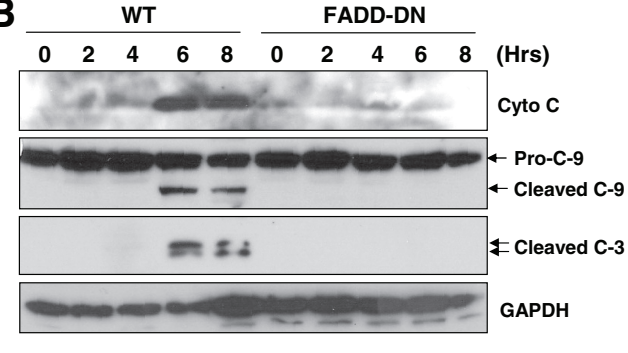

D
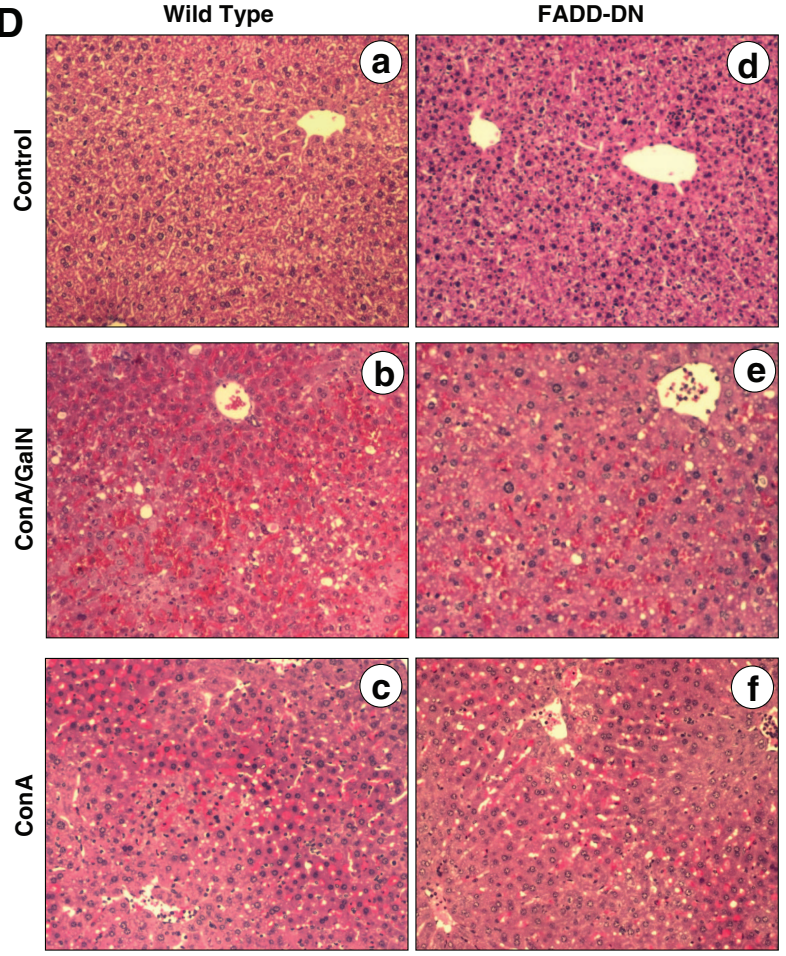

the role of apoptosis in these two regimes by using a transgenic mouse strain that expresses a liver-specific truncated form of FADD (FADD-DN), which exerts a dominant negative effect by competing with the wild-type (WT) FADD. ${ }^{20}$ Significant caspase activation in the liver tissue could be detected in WT mice, but not in FADD-DN mice, treated with ConA/GalN (Figure 1, A-B). Previous studies have indicated that hepatocytes are Type II cells in which the death receptor-initiated apoptosis requires the mitochondrial participation. ${ }^{4,6,7}$ Consistently, the mitochondrial pathway was inhibited in FADD-DN livers with no cytochrome c release and caspase-9 activation (Figure 1B). These observations indicate that ConA/GalN could induce a potent mitochondrial apoptotic response and caspase-activation in a FADD-dependent manner.

Surprisingly, liver injury as measured by the serum level of ALT was not obviously affected by FADD-DN in ConA/GalN-treated mice (Figure 1C). Histological evaluation indicated significant hepatocyte apoptosis, loss of cellular integrity and massive red cell infiltration into the parenchyma in ConA/GalN-treated WT livers, which were not seen in FADD-DN livers (Figure 1D). However, histological changes characterized by increased eo- 
A

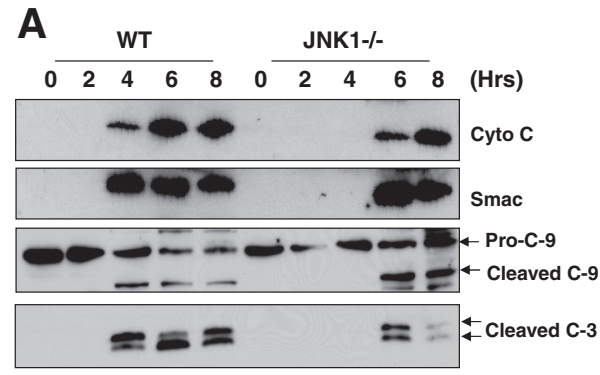

\section{B}

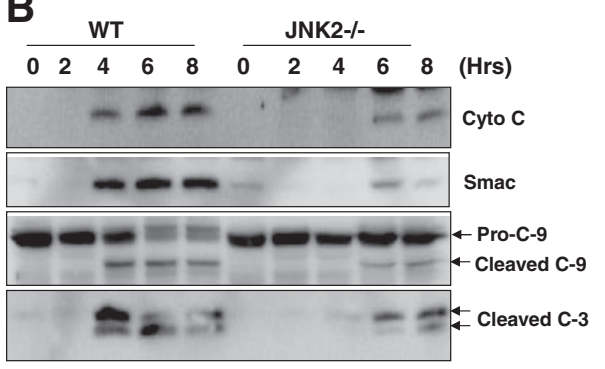

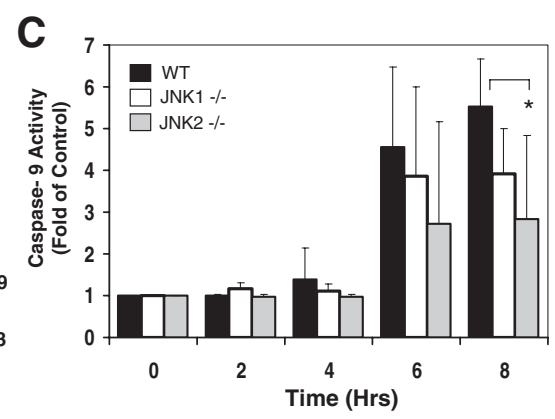

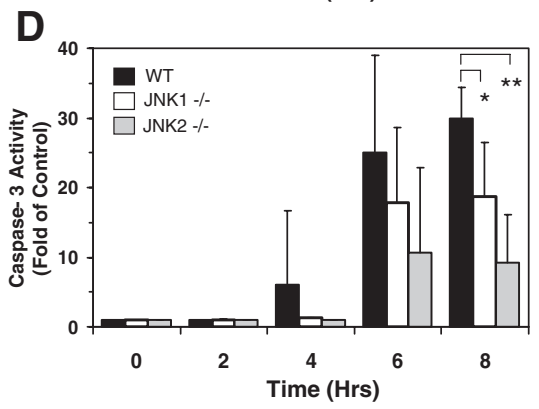

Figure 2. JNK1-deficient (JNK1-/-) and JNK2-deficient (JNK2-/-) mice have reduced mitochondrial apoptotic response on ConA/ GalN treatment. A-B: WT, JNK1-/- (A) and JNK2 $-/-$ mice $(\mathbf{B})$ were treated with ConA/ GalN and sacrificed at the designated time point. Liver lysates were prepared for immunoblot analysis with the indicated antibodies (molecular weight of Smac is $25 \mathrm{kDa}$ ). C-D: Liver cytosols of WT (black columns), JNK1-/- (white columns), and JNK2-/- (gray columns) mice treated as above were analyzed for caspase- 9 (C) and -3 (D) activities. Results (mean \pm SD) are expressed as the fold of the non-treated control. Groups that had significantly different responses are indicated $\left({ }^{*} P<0.05 ;{ }^{* * *} P<0.01\right)$. sinophilic staining, hepatocyte swelling and minor accumulation of red cells in the sinusoids could be still observed in the FADD-DN livers. A semiquantitative assessment indicated a composite injury score of $8 \pm 0$ for the WT livers and $4 \pm 0$ for the FADD-DN livers, whereas the scores for the untreated livers would be zero (see Experimental Procedures for scoring criteria). These findings suggested that there was also a FADD-independent non-apoptotic liver injury induced by ConA/GalN.

In contrast to the ConA/GalN regime, no caspase-8, -9, and -3 activities could be detected in WT mouse livers following the treatment with ConA for 8 hours (Figure 1A) and there was no mitochondrial apoptotic activation (see below). Inhibition of FADD made no difference as expected. Nevertheless, serum ALT level was significantly elevated in both strains of mice (Figure 1C). Histological examination of the liver demonstrated obvious cellular swelling, increased eosinophilic staining and red cell accumulation in the sinusoids with occasional cellular vacuolization and cell lysis (Figure 1D). The histological features between the WT and FADD-DN in the ConA regime were similar although they were slightly less significant in the latter (a composite score of $5 \pm 0$ and $4 \pm 1$ for the WT and FADD-DN livers, respectively). Interestingly, these features were also similar to those observed in the FADD-DN mice treated with ConA/GalN and there were no significant differences in the severity of the injury (a score of $4 \pm 1$ and $4 \pm 0$ for ConA alone and ConA/GalN, respectively, see above). Taken together, these results indicated that ConA primarily induced a FADD-independent necrotic injury, whereas ConA/GalN induced not only FADD-independent necrotic injury, but also FADD-dependent apoptotic injury.

\section{JNK Participates in ConA/GalN-Induced Mitochondrial Apoptosis in the Liver}

We then examined how JNK could contribute to TNF $\alpha$ induced liver injury in the two ConA regimes. Deletion of either JNK1 or JNK2 led to reduced and delayed mitochondrial release of cytochrome $\mathrm{c}$ and Smac, and caspase activation in the liver on ConA/GalN treatment (Figure 2, A-D). The apoptotic response could be detected at 4 hours after treatment in the WT mice, but were delayed in the JNK1 or JNK2-deficient mice. In addition, the response level was much reduced, particularly in JNK2-deficient mice.

JNK1 and JNK2 have a major isoform of $46 \mathrm{kDa}$ and 54 $\mathrm{kDa}$, respectively (Figure $3, \mathrm{~A}-\mathrm{B}$ ). Both have a minor isoform as well, ie, the p46 JNK2 and p54 JNK1. ${ }^{24}$ All these forms could be persistently phosphorylated following ConA/GalN treatment (Figure 3, A-B). However, it seemed that the p54 form of JNK2 was likely the major phosphorylation species, as deletion of JNK2 seemed to affect this phosphorylation more than the deletion of JNK1. p46 JNK1 could be phosphorylated as well, which became obvious in the JNK2-deficient liver, perhaps due to the elimination of the futile phosphorylation of JNK2 as suggested previously. ${ }^{25}$ Notably, it seemed that JNK1 was responsible for the C-Jun kinase activity after ConA/ GalN treatment, whereas JNK2 was dispensable (Figure 3, C-D). Similar findings had been observed previously in fibroblasts. ${ }^{25}$ These results suggest that JNK phosphorylation and the c-Jun kinase activity of JNK are not coupled.

Since TNF $\alpha$-induced mitochondrial activation is related to caspase- 8 activation and Bid cleavage, we investigated the mechanism of JNK in promoting ConA/GalNinduced apoptosis by examining these events. Caspase-8 was cleaved around 4 hours, and reached peak around 6 


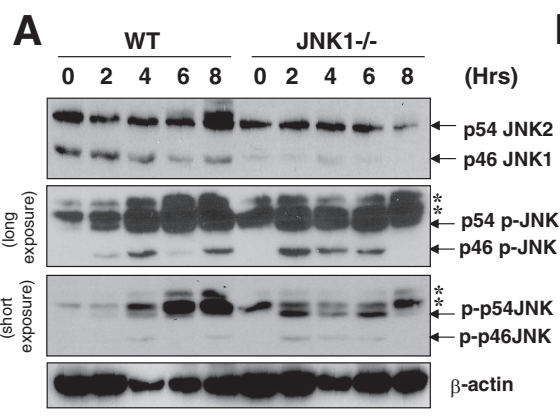

B

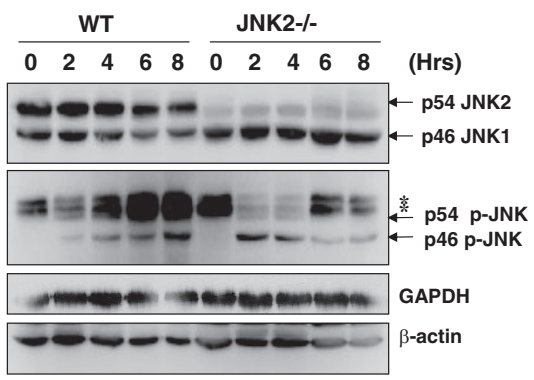

C

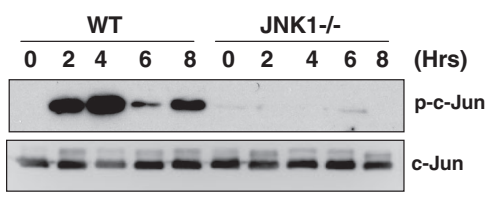

D

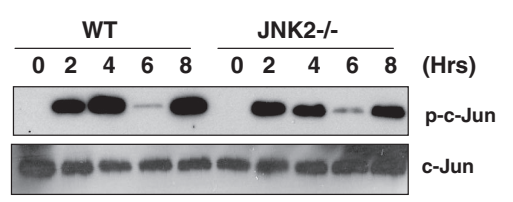

Figure 3. Both JNK1 and JNK2 are phosphorylated but only JNK1 exhibits c-Jun kinase activity on ConA/GalN treatment. A-B: Mice were treated and liver lysates were prepared as in Figure 2 Immunoblot assay was conducted using antibodies against phosphorylated JNK (p-JNK) and total JNK. Note that the majority of JNK1 are $46 \mathrm{kDa}$ and the majority of JNK2 are $54 \mathrm{KD}$ as indicated. The minor band around $46 \mathrm{kDa}$ seen in JNK1 $-/-$ liver represents the p46 isoform of JNK2. Conversely, the minor band around $54 \mathrm{kDa}$ seen in JNK2-/liver represents the p54 isoform of JNK1. Phosphorylated JNK could be both the major and the minor forms of JNK1 or JNK2 and are thus not specified other than the molecular weight. $\beta$-actin is $46 \mathrm{kDa}$. Asterisks indicated non-specific bands. C-D: The same cytosolic fraction was used for the in vitro JNK kinase assay with GST-C-Jun as the substrate. Phosphorylation of c-Jun by JNK was determined using the antibody against phosphorylated c-Jun (p-c-Jun, $35 \mathrm{kDa}$ ). The same membranes were immunoblotted with an anti-c-Jun antibody to confirm the protein loading. to 8 hours after ConA/GalN treatment as indicated by the reduction of the pro-caspase-8 (Figure 4, A-B). This correlated well with the kinetics of the elevation of casapase-8 activity (Figure 4C) and cleavage of Bid (Figure 4, A-B). However, caspase-8 activation was clearly reduced and Bid cleavage was almost eliminated in the absence of JNK1 or JNK2 (Figure 4, A-B). In addition, it seemed that while FLIP $\mathrm{L}_{\mathrm{L}}$ was consistently degraded in the WT livers, it remained relatively stable in the JNK1 or JNK2-deficient livers (Figure 4, A-B). Since FLIP $P_{L}$ is a key negative regulator of caspase- 8 activation, the relative stabilization of $F L I P_{L}$ may in part explain the weakened caspase-8 activation in the JNK1 or JNK2deficient livers.

Since ConA activates $T$ cells, which in turn produce a number of cytokines including $\mathrm{TNF} \alpha$ and $\mathrm{IFN} \gamma$, which cause liver injury, ${ }^{26-28}$ we examined the serum TNF $\alpha$ and IFN $\gamma$ levels in JNK-deficient mice. To determine whether JNK may affect liver injury by regulating $T$ cell response, we found that IFN $\gamma$ levels were not significantly different between the WT and JNK1 or JNK2-deficient mice (Figure $5 \mathrm{~A}$ ). On the other hand, TNF $\alpha$ level seemed to be increased in JNK1-deficient mice at 2 hours after treatment, but was reduced in JNK2-deficient mice at both 2 and 4 hours (Figure 5A). Although statistic difference was not established here these changes could potentially affect the severity of the liver injury seen in these mice.

Conversely, to determine whether JNK could play the pro-apoptotic role in the liver, we established chimeric mice via bone marrow transplantation. Since JNK2-deficient mice seemed to be more resistant to ConA/GalN treatment, we examined this issue based on the transplantation of WT bone marrow nucleated cells to lethally irradiated JNK2-deficient mice as well as to WT mice. Three months later, the transplanted cells were established in the recipient mice as indicated by flow cytometry analysis of the splenic lymphocyte populations. There were no significant differences in the total splenic cell numbers (data not shown) and in the percentage of splenic $T$ and $B$ cell subpopulations between the two groups of reconstituted mice and the native WT mice (Figure 5B). Immunoblot analysis using the anti-JNK antibody confirmed that the reconstituted splenocytes in the JNK2-deficient mice were derived from the WT mice, whereas the liver remained JNK2-deficient (Figure 5C). When challenged with ConA/GalN, the WT or JNK2-deficient mice reconstituted with WT bone marrows had noticeably decreased mitochondrial apoptotic response and caspase activation, compared to the corresponding non-reconstituted mice (Figure 5, D-E), perhaps due to
A

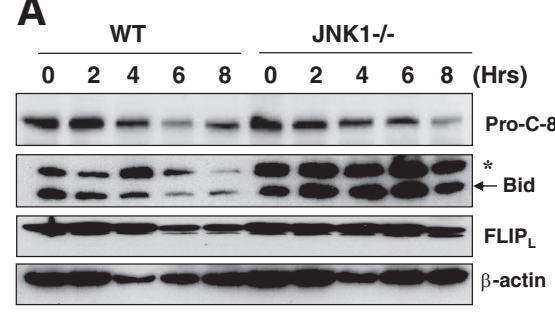

B

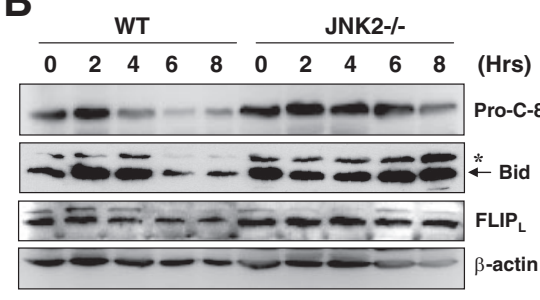

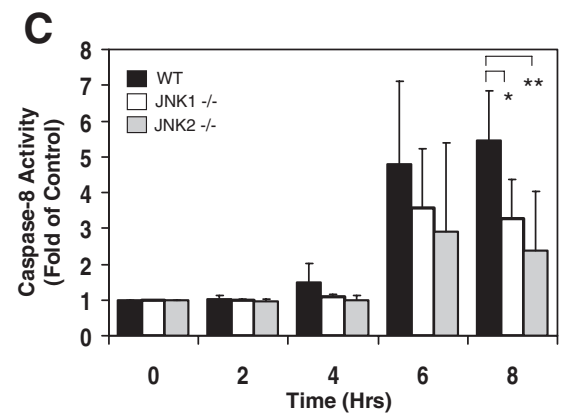

C

Figure 4. JNK1 and JNK2 play an important role in promoting caspase- 8 and Bid activation. A-B: Mice were treated and liver lysates were prepared as in Figure 2. Immunoblot assay was conducted with indicated antibodies (pro-c-8: p 57 pro-caspase-8, p21 Bid, p55 FLIP ${ }_{\mathrm{L}}$, and p46 $\beta$-actin). Asterisks indicated non-specific bands. C: Cytosolic fractions of the liver lysates from WT (black columns), JNK1-/- (white columns), and JNK2-/- (gray columns) mice treated as above were analyzed for caspase- 8 activities. Results (mean \pm SD) are expressed as fold of changes over the untreated control. Groups that had significantly different responses are indicated $\left({ }^{*} P<0.05 ;{ }^{* *} P<0.01\right)$. 
A
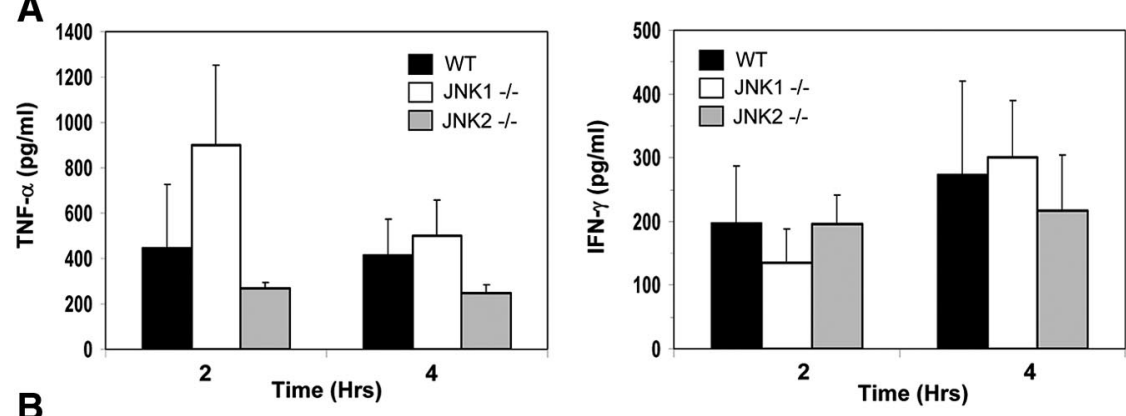

B
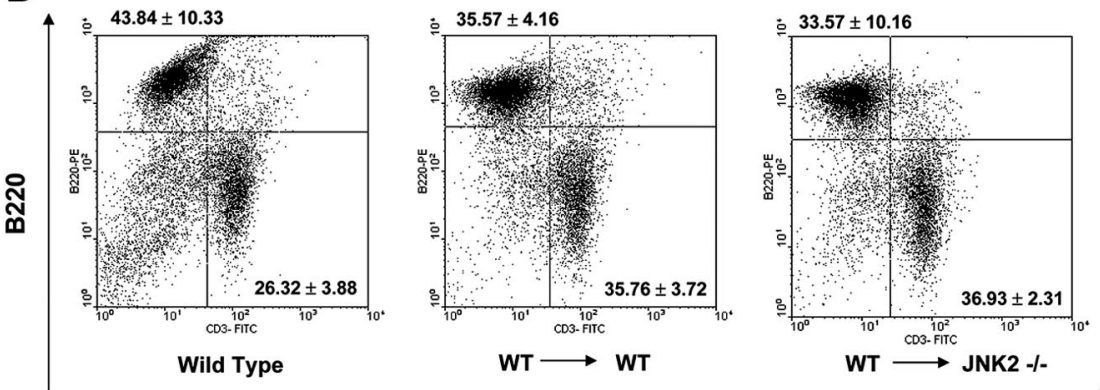

CD3

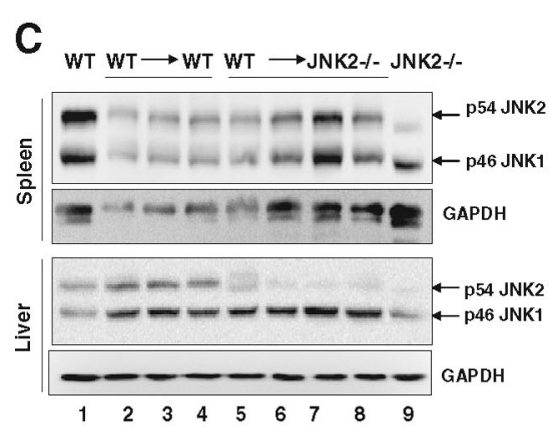

D

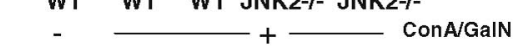

WT $\quad$ WT $\quad \underset{\mathrm{WT}}{\overrightarrow{\mathrm{JNK}} 2-I-\mathrm{JNK} 2-- \text { - }}$

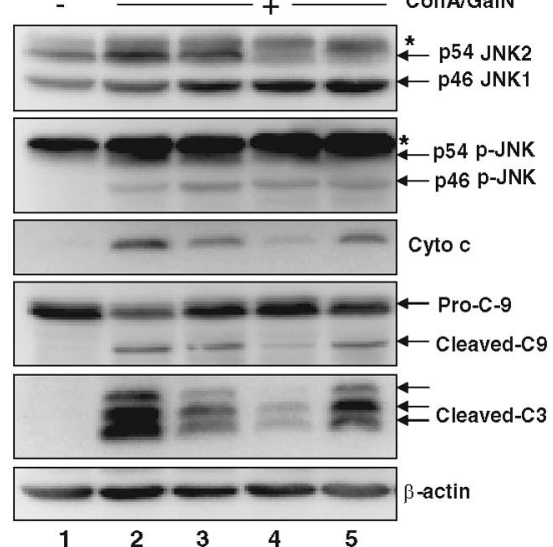

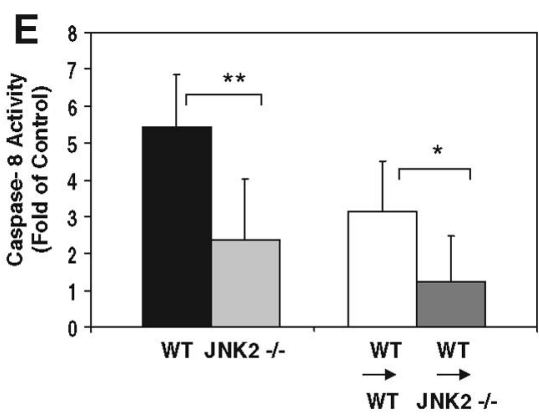
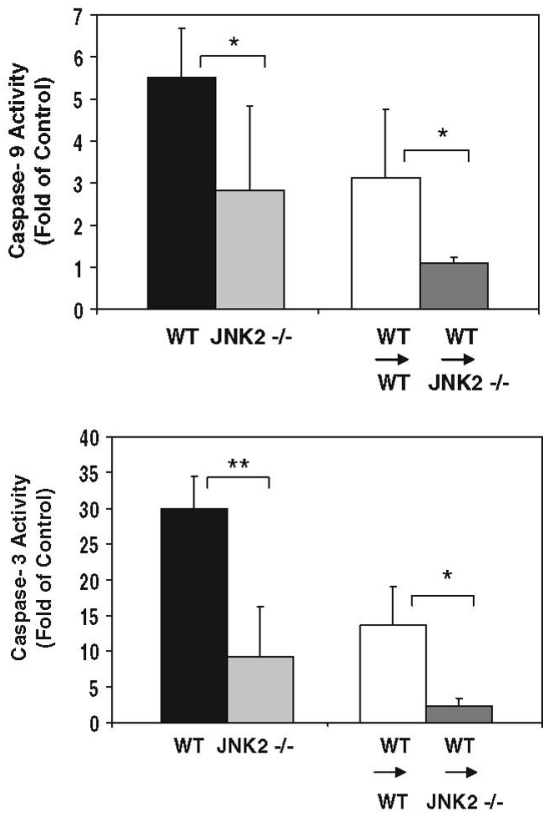

Figure 5. JNK2-deficient (JNK2-/-) livers are protected from ConA/GalN-induced apoptosis. A: WT (black columns), JNK1-/- (white columns), and JNK2 $-/-$ (gray columns) mice were treated with ConA/GalN and sacrificed at the designated time point. Serum levels of TNF- $\alpha$ and IFN- $\gamma$ were determined. Data (mean $\pm \mathrm{SD}$ ) are from at least three mice for each group at the indicated time points. There are no statistical differences between WT and the JNK1 or JNK2-deficient mice. B: Splenocytes recovered from normal WT mice (left panel, $n=4$ ), or WT mice reconstituted with WT bone marrow (WT $\rightarrow$ WT, middle panel, $n=4$ ), or JNK2 $-/-$ mice reconstituted with WT bone marrow (WT $\rightarrow$ JNK2 $-/-$, right panel, $n=5$ ) were analyzed by flow cytometry using fluorescein isothiocyanate-anti-CD3 and PE-anti-B220. The percentages (mean \pm SD) of B220-positive cells (B cells) and CD3-positive cells (T cells) are indicated at the upper left quadrant, and lower right quadrant, respectively. C: Spleen lysates and liver cytosols from the above mice, and JNK2-deficient (JNK2-/-) mice were analyzed by immunoblot assay with antibodies against total JNK and GAPDH. Each lane represents one mouse sample. D: Liver cytosols from the same group of mice as in $\mathrm{C}$ after 8 hours of ConA/GalN treatment were subjected to immunoblot assay with the indicated antibodies. Asterisks indicated non-specific bands. Note the differences between lanes 2 and 5, and between lanes 3 and 4 . E: The liver cytosolic fractions from ConA/GalN-treated mice were analyzed for caspase-8, -9, and -3 activities (WT $n=7$, JNK2 $-/-n=7$, WT $\rightarrow$ WT $n=8$, WT $\rightarrow$ JNK2 $-/-n=9$ ). Results (mean \pm SD) are expressed as fold of the changes over the untreated controls. Groups that had significantly different responses are indicated $\left({ }^{*} P<0.05 ;{ }^{* *} P<0.01\right)$.

an overall low immune capability in the reconstituted mice at this stage. However, the reconstituted JNK2deficient mice consistently had a lower apoptotic response than that of the reconstituted WT mice (Figure 5, D-E). Since these mice were reconstituted with the same WT bone marrows, this result indicated that the difference between the two strains were due to the difference in JNK2 expression in the liver and thus confirmed the role of JNK2 in the apoptotic response in the liver.

\section{Differential Roles of JNK in Liver Injury Caused by ConA/GalN and that by ConA}

ConA alone at the dose of $25 \mathrm{mg} / \mathrm{kg}$ does not cause lethal liver injury. Together with GalN, this dose of ConA is lethal with most of the mice died around 6 to 8 hours. Deletion of JNK1 or JNK2 significantly reduced the mortality. While the mean survival time for the WT mice were $7.45 \pm 0.35$ hours, those for JNK1-deficient and JNK2-deficient mice 
A
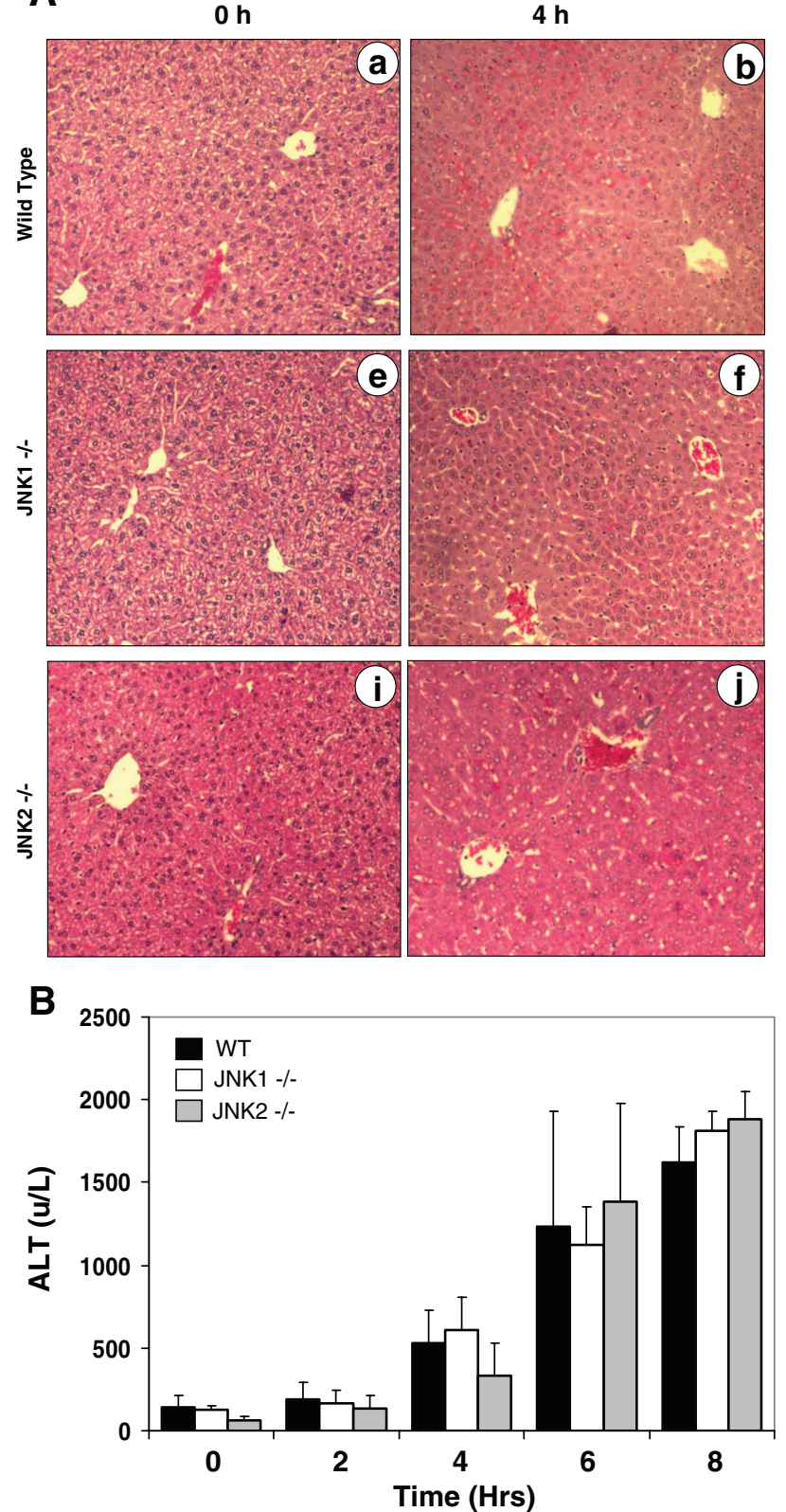
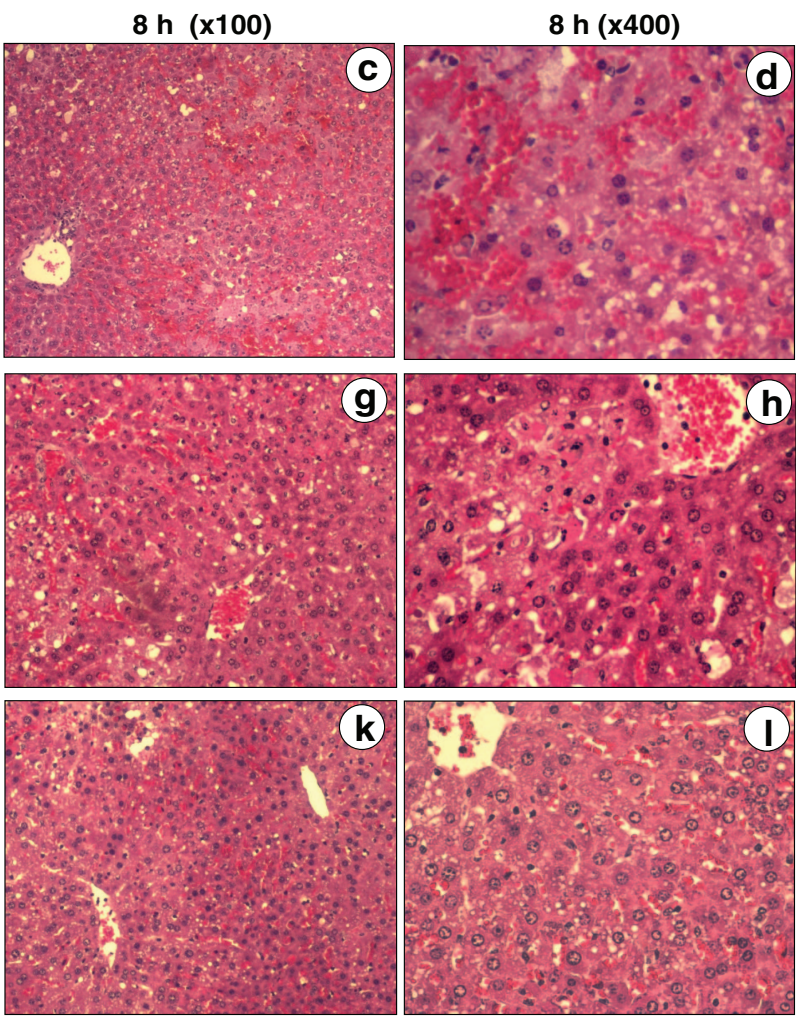

\section{C}

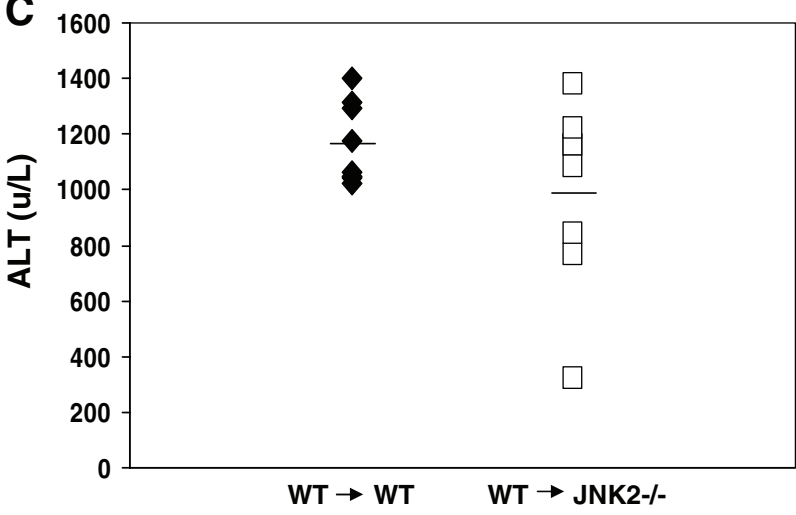

Figure 6. JNK1 and JNK2-deficient mice remain sensitive to ConA/GalN-induced liver injury. A: Sections of livers from WT (a-d), JNK1 - / - (e-h), or JNK2-/(i-l) mice treated with ConA/GalN for $0(\mathrm{a}, \mathrm{e}, \mathrm{i}), 4(\mathrm{~b}, \mathrm{f}, \mathrm{j})$, or $8(\mathrm{c}-\mathrm{d}, \mathrm{g}-\mathrm{h}, \mathrm{k}-\mathrm{l})$ hours were H\&E stained and examined at a lower power $(\times 100)(\mathrm{a}-\mathrm{c}, \mathrm{e}-\mathrm{g}$, i-k) or at a higher power $(\times 400)(\mathrm{d}, \mathrm{h}, 1)$. B: WT (black columns), JNK1 $-/-$ (white columns), and JNK2-/- (gray columns) mice were treated with ConA/GalN and sacrificed at the designated time point. Serum ALT levels were measured. Results (mean \pm SD) are from at least three mice for each group at the indicated time points. There are no statistical differences among the groups. C: WT mice reconstituted with WT bone marrow (WT $\rightarrow$ WT, $n=8$ ), or JNK2 $-/-$ mice reconstituted with WT bone marrow (WT $\rightarrow$ JNK $2-/-, n=8$ ) were treated with ConA/GalN for 8 hours. Serum ALT levels (mean \pm SD) were determined. Each data point represents one sample. The horizontal bars indicate the mean values. There are no statistical differences between the two groups.

were $11.08 \pm 1.96$ hours and $13.38 \pm 1.89$ hours, respectively. The massive apoptosis, cell lysis, blood vessel destruction and parenchymal alterations as seen in the WT mice were inhibited in the JNK1-deficient and JNK2-deficient mice (Figure 6A). Semiquantitative analysis of the histological changes indicated a composite score of $8 \pm 0,4.5 \pm 0.7$, and $2.7 \pm 0.6$ for the WT, JNK1-deficient and JNK2-deficient livers, respectively. The somewhat stronger protection of JNK2 deficiency was consistent with the more significant effect of this deficiency in suppressing mitochondria apoptotic re- sponse and caspase activation (Figures 2 and 4). Taken together these observations supported that JNK1 and JNK2 participated in the hepatic apoptosis program triggered by ConA/GalN and promoted the lethal injury.

Surprisingly, the serum ALT levels remained high at the later time point (6 to 8 hours) in the ConA/GalN-treated JNK-deficient mice either at the native condition (Figure $6 \mathrm{~B}$ ) or in the bone marrow chimeric status (Figure 6C). Only moderate reduction, mainly in the JNK2-deficient mice and/or in early time point could be observed. Consistently, these mice retained histological changes that 
A

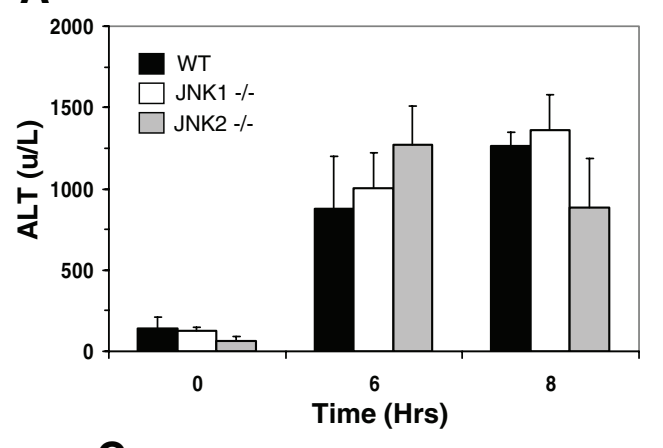

C

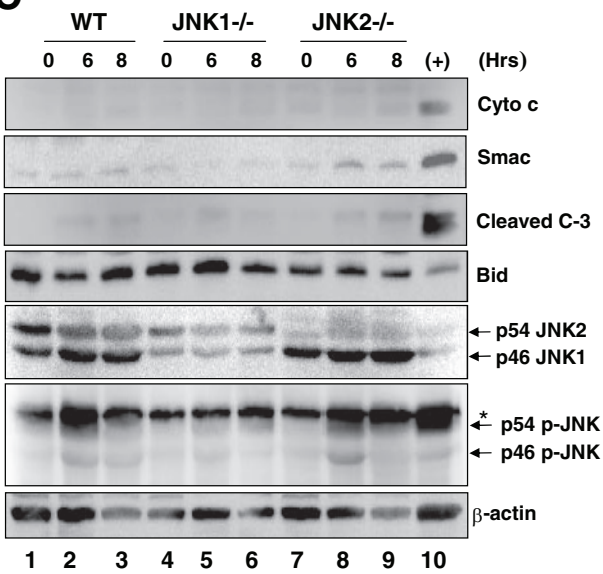

D

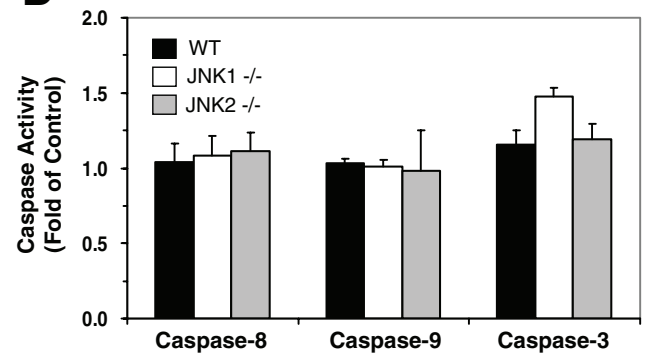

were similar to those seen in ConA/GalN-treated FADD-DN liver, ie, hepatocyte swelling and red cell accumulation in the sinusoids (Figures 6A and 1D). These observations thus suggest that like FADD, JNK may not play a major role in the non-apoptotic injury caused by ConA/GalN.

To further examine this issue, we treated JNK1- and JNK2-deficient mice with ConA alone, which, based on above studies, should cause a non-apoptotic injury. We detected no significant reduction in ALT levels in JNK1 or JNK2-deficient mice treated with ConA alone (Figure 7A), compared to the WT mice. In addition, there were no differences in the histological manifestations between the WT and JNK-deficient mice (Figure 7B), although there were some reductions in the extent of the injury (an injury score of $5 \pm 0$ and $3.3 \pm 0.6$ for the WT and JNK2deficient livers, respectively). Notably, the histological presentations were similar to those observed in FADD-DN mice receiving ConA alone, and furthermore, were similar to those observed in FADD-DN and JNK-
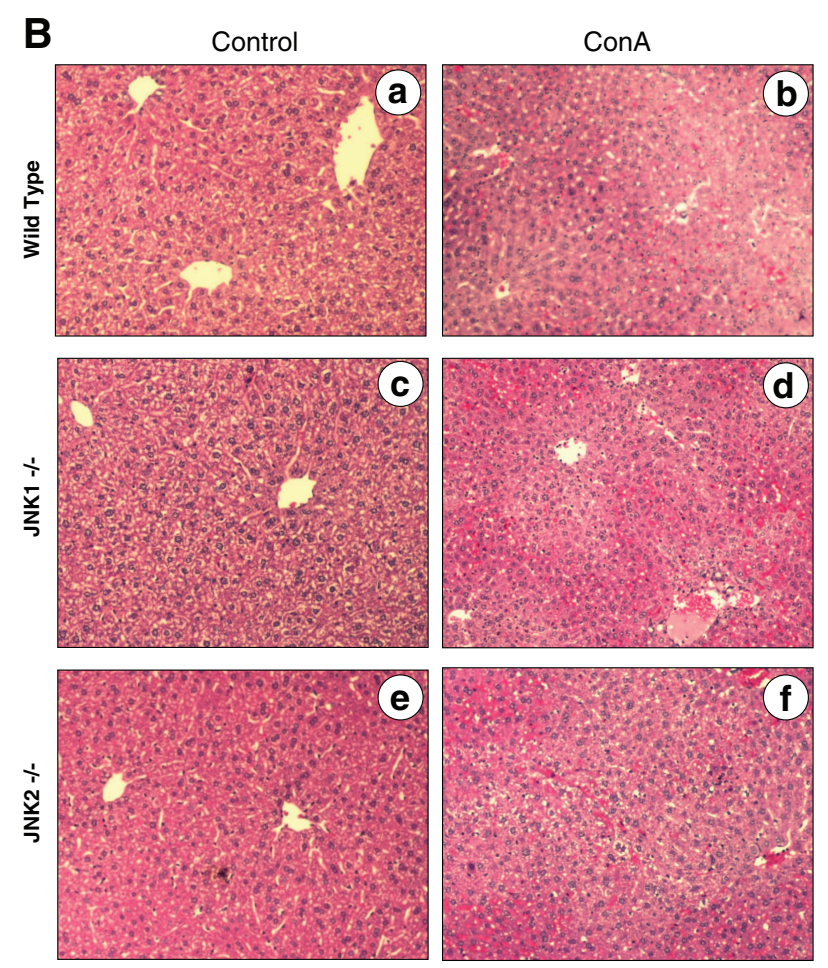

Figure 7. Deletion of JNK1 or JNK2 does not affect ConA-induced nonapoptotic liver injury. A: Serum ALT levels from WT, JNK1 -/- or JNK2-/mice treated with ConA for 6 or 8 hours were measured (mean \pm SD). There are no statistical differences between the WT and the JNK1 or the JNK2deficient mice. B: Sections of livers from WT (a-b), JNK1-/- $(c-d)$, or JNK $2-/-(\mathrm{e}-\mathrm{f})$ mice treated with ConA $(\mathrm{b}, \mathrm{d}, \mathrm{f})$ for 8 hours or untreated (a, c, e) were H\&E stained. C: WT, JNK1 $-/-$, or JNK2 $-/-$ mice were treated with ConA and sacrificed at 6 or 8 hours later. Liver cytosols were subjected to the immunoblot assay with the indicated antibodies. Asterisks indicated non-specific bands. Lane 10 represents a sample of WT mouse treated with ConA/GalN for 8 hours. D: The same lysates as those used in (B) were examined for caspase activities using the corresponding substrates. Results (mean $\pm \mathrm{SD})$ are expressed as fold of changes over the non-treated control.

deficient mice treated with ConA/GalN (Figures 1D and $6 \mathrm{~A})$. Consistently, there were no activation of mitochondrial apoptosis pathway (Figure 7C) and no increases in caspase-activity (Figure 7D) in ConA-treated WT, JNK1deficient, or JNK2-deficient livers. Taken together these data indicated that JNK1 or JNK2 was involved in ConA/ GalN-induced apoptotic liver injury but not in ConA-induced non-apoptosis-mediated liver injury.

\section{Discussion}

\section{Different Modes of Cell Death in ConA and ConA/GalN Regimes}

$\mathrm{T}$ cell mediated liver injury can be commonly analyzed following an intravenous injection of a $T$ cell mitogen, ConA, with or without a pre-sensitization by GalN. Cytokines play a significant role in the development of liver injury. ${ }^{26-28}$ Although in both cases, $\mathrm{TNF} \alpha$, generated by 
T cells, is the main cytokine that causes the injury, ${ }^{26}$ there are significant differences between the two models. Specifically, caspase-dependent apoptosis is the dominant mode of cell death in ConA/GalN model, but not in the ConA model. As such the injury is usually irreversible in the former, but reversible in the latter.

While ConA-induced liver injury depends on both TNF-R1 and TNF-R2, caspase activation in ConA/GalN treatment depends only on TNF-R $1 .{ }^{13}$ Early work had also shown that the liver injury could be greatly inhibited by a pan-caspase inhibitor in the ConA/GalN model, but not in the ConA only model. ${ }^{13}$ TNF $\alpha$-R1-mediated caspase activation depends on the adaptor molecule TNF receptor-associated death domain and FADD, which recruit caspase-8. Consistently, we found that FADD-DN mice were well protected from ConA/GalN injury with no caspase activation. These mice had been shown before to be resistant to anti-Fas and LPS/GalN-induced liver injury. ${ }^{20}$ It is thus predictable that ConA/GalN-induced apoptosis in the hepatocyte would be dependent on mitochondria as in the other models of death receptorinitiated liver injury. Indeed, mitochondrial release of cytochrome c could be detected early on in WT mice, but was not observed in the FADD-DN mice. The mitochondria response would be mediated by Bid, which is translocated to the mitochondria following the cleavage by caspase-8. We did find a significant reduction of mitochondria apoptosis in the Bid-deficient mice following ConA/GalN treatment (data not shown), as in the anti-Fas and LPS/GalN treatment. $4,6,7$ Thus suppression of caspase-8 activation by FLIP, an NF- $\kappa$ B target, is a crucial regulatory mechanism.

No apparent caspase activation could be detected in the absence of GalN at the time when the liver enzymes had already arisen significantly in the blood (Figures 1 and 7 ), which was also observed in early studies. ${ }^{14,13}$ The injury could clearly occur in a caspase-independent way, as they could be also observed in the FADD-DN mice (this study) and could not be suppressed by blocking antibodies against FADD/caspase 8 cascade, ${ }^{28}$ or by a pan-caspase inhibitor. ${ }^{13}$ ConA alone thus induced a mainly non-caspase dependent cell death, which seems to be oncotic necrosis. While the exact mechanisms of TNF $\alpha$-induced necrotic mechanisms are not clear, receptor-interacting protein-mediated pathway and reactive oxygen species generation have been implicated. ${ }^{9,10}$ The latter could be linked to the activation of mitochondria permeability transition pore and reduction of ATP production, which could contribute to necrosis. ${ }^{29}$ Interestingly, despite that hepatocytes can mount a significant apoptosis in response to ConA stimulation in the presence of GalN, the necrotic death still occurs, as observed in FADD-DN mice. In these mice, while apoptosis was completely blocked following ConA/GalN treatment, blood liver enzyme level was still elevated and liver histology was still abnormal. Both presentations resembled to what could be observed in the ConA model. These observations support the notion that the apoptotic death and non-apoptotic death in the two ConA models are clearly mediated by different signal pathways. While both modes of cell death are activated in the ConA/GalN model, only the necrotic mode is activated in the ConA alone mode. It seems that the default mechanism of cell death in the ConA regime is necrosis and apoptosis is induced to appreciable levels only when GalN is applied and when the apoptosis pathways are intact.

\section{Differential Role of JNK in ConA Versus ConA GalN-Induced Liver Injury}

JNK has been widely implicated in TNF $\alpha$-induced toxicity in hepatocyte. ${ }^{17-19,23} \mathrm{JNK}$, particularly JNK2, was found to be critical to hepatocyte apoptosis and liver injury in the LPS/GalN regime ${ }^{19}$ and in the ischemia/reperfusion regime. ${ }^{30}$ Here we have demonstrated that JNK is important for the ConA/GalN-induced injury but not ConA-induced injury. In the formal, JNK2 seems to play a more prominent role than JNK1. The bone marrow transplantation study indicated that the pro-death effect of JNK could be manifested at the liver level.

JNK may promote cell death by different mechanisms in different cells. Several studies now indicate that JNK is involved in the mitochondria activation. ${ }^{17-19,23,31}$ This seems to be the case in the ConA/GalN model, in which deficiency of JNK1 or JNK2 greatly reduced mitochondria activation and caspase activation. It seems that JNK affects mitochondria activation by controlling the upstream events. Therefore degradation of $F L I P L$ was blocked in the absence of JNK and in turn caspase-8 activation was reduced with decreased cleavage of Bid. These observations are consistent with previous findings that TNF $\alpha$-induced caspase cascade can be blocked by FLIP, ${ }^{32}$ and that FLIP $\mathrm{L}$ degrades following TNF $\alpha$ stimulation $^{33}$ through a JNK-mediated activation of its E3 ligase, Itch. ${ }^{34}$

Several additional observations need to be considered for the role of JNK in the liver injury scenario. First, it seemed that neither the phosphorylation status nor the c-Jun kinase activity is closely linked with the pro-death activity, although they were good indications of JNK activation. This discord had also been observed in the liver injury induced by LPS/GaIN. ${ }^{19}$ The C-Jun kinase activity seems to be mostly associated with JNK1, which was intact in the JNK2-deficient mice. This was consistent with what had been reported in fibroblasts ${ }^{25}$ and other cells. ${ }^{35}$ However, unlike in the fibroblasts, ${ }^{25}$ JNK2-deficient mice conferred a greater protection against liver injury than JNK1-deficient mice. The c-Jun kinase activity of JNK2, although much lower than that of JNK1, may still be contributory to apoptosis induction. In addition, the ability of JNK2 to promote c-Jun degradation and therefore to suppress cell proliferation ${ }^{35}$ may in turn antagonize the pro-survival status of the cell. Alternatively, other yet-to-be-defined activities of JNK2 could be instrumental. From this aspect, it is possible that the ability of JNK to activate Itch could be segregated from its ability to activate c-Jun. These possibilities have yet to be examined in future studies. Finally, it should be noted that JNK could promote mitochondria activation via non-Bid-mediated pathways. ${ }^{23,36}$ 
In contrast to what was observed in the ConA/GalN model, we did not observe any significant protection against ConA-induced injury conferred by the deletion of JNK1 or JNK2 as measured by serum ALT level and liver pathology. Similar observation was made when a chemical JNK inhibitor was used. ${ }^{37}$ This was not the case in a previously reported study in that JNK1 or JNK2-deficient mice were protected. ${ }^{15}$ The reason for this discrepancy is not clear. One possible explanation may be related to the interpretation of certain parameters, such as terminal deoxynucleotidyl transferase-mediated dUTP nick-end labeling staining, which was not used in present study because it was not a reliable marker for apoptosis and it could be misleading without a careful quantification and other supportive evidence.

However, it may not be surprising that JNK does not participate in ConA-mediated liver injury, as it was mainly caused by non-apoptotic death, as discussed above. Most evidence supports that JNK participates in the apoptotic response in TNF $\alpha$-induced toxicity. In the ConAinduced injury, apoptosis is only present when GalN is given, which blocks NF- $\kappa$ B activation. A major function of $\mathrm{NF}-\kappa \mathrm{B}-$ mediated protection is to suppress sustained JNK activation, which is required for its pro-death effect. ${ }^{16,38-41}$ Indeed, sustained JNK activation is most clearly seen when NF- $\kappa \mathrm{B}$ is blocked in the ConA/GalN treatment (Figure $3 \mathrm{~A}$ ). Thus JNK is important in ConA/GalN-induced injury likely because of the apoptotic nature of the injury and the suppression of NF- $\kappa \mathrm{B}$. Conversely, JNK does not seem to be important in ConA-mediated injury likely because of its non-apoptotic nature of the injury and because there is no suppression of NF- $\kappa \mathrm{B}$. Consistently, in other types of cells in which TNF $\alpha$-induced non-apoptotic death was documented, JNK was also found to be nonessential. ${ }^{9,10}$ It has to be pointed out that JNK can participate in non-apoptotic death in some non-TNF $\alpha$-mediated scenarios. For example, JNK is critical for acetaminophen-induced liver injury, which is characterized by caspase-independent oncotic necrosis. ${ }^{37}$ This effect of JNK is specific to this type of injury and was not observed in injury caused by $\mathrm{CCl}_{4}$ or ConA. ${ }^{37}$

In summary, ConA stimulation of liver injury evokes different cell death modes depending on the presence of the sensitizing agent, GalN. Whereas ConA alone mainly induce a caspase-independent necrotic death and mild reversible liver injury, ConA/GalN also activates a potent caspase-dependent apoptotic response, in which JNK participates to promote mitochondria activation, leading to irreversible liver injury and mortality.

\section{Acknowledgment}

We thank Dr. Razq Hakem (University of Toronto, Canada) for the anti-caspase-8 antibody.

\section{References}

1. Wajant $H$, Pfizenmaier $K$, Scheurich $P$ : Tumor necrosis factor signaling. Cell Death Differ 2003, 10:45-65

2. Ding WX, Yin XM: Dissection of the multiple mechanisms of TNF- alpha-induced apoptosis in liver injury. J Cell Mol Med 2004, 8:445-454

3. Schwabe RF, Brenner DA: Mechanisms of liver injury. I. TNF-alphainduced liver injury: role of IKK, JNK, and ROS pathways. Am J Physiol Gastrointest Liver Physiol 2006, 290:G583-G589

4. Li L, Thomas RM, Suzuki H, De Brabander JK, Wang X, Harran PG: A small molecule Smac mimic potentiates TRAIL- and TNF\{alpha\}-mediated cell death. Science 2004, 305:1471-1474

5. Eckelman BP, Salvesen GS, Scott FL: Human inhibitor of apoptosis proteins: why XIAP is the black sheep of the family. EMBO Rep 2006 , 7:988-994

6. Yin XM, Wang K, Gross A, Zhao Y, Zinkel S, Klocke B, Roth KA, Korsmeyer SJ: Bid-deficient mice are resistant to Fas-induced hepatocellular apoptosis. Nature 1999, 400:886-891

7. Zhao Y, Li S, Childs EE, Kuharsky DK, Yin X-M: Activation of pro-death $\mathrm{Bcl}-2$ family proteins and mitochondria apoptosis pathway in tumor necrosis factor-alpha -induced liver injury. J Biol Chem 2001, 276:27432-27440

8. Du C, Fang M, Li Y, Li L, Wang X: Smac, a mitochondrial protein that promotes cytochrome c-dependent caspase activation by eliminating IAP inhibition. Cell 2000, 102:33-42

9. Lin Y, Choksi S, Shen H-M, Yang Q-F, Hur GM, Kim YS, Tran JH, Nedospasov SA, Liu Z-G: Tumor necrosis factor-induced nonapoptotic cell death requires receptor-interacting protein-mediated cellular reactive oxygen species accumulation. J Biol Chem 2004, 279: 10822-10828

10. Byun HS, Park KA, Won M, Yang K-J, Shin S, Piao L, Kwak JY, Lee Z-W, Park J, Seok JH, Liu Z-G, Hur GM: Phorbol 12-myristate 13acetate protects against tumor necrosis factor (TNF)-induced necrotic cell death by modulating the recruitment of TNF receptor 1 -associated death domain and receptor-interacting protein into the TNF receptor 1 signaling complex: implication for the regulatory role of protein kinase C. Mol Pharmacol 2006, 70:1099-1108

11. Trautwein C, Rakemann T, Brenner DA, Streetz K, Licato L, Manns MP, Tiegs G: Concanavalin A-induced liver cell damage: activation of intracellular pathways triggered by tumor necrosis factor in mice. Gastroenterology 1998, 114:1035-1045

12. Trautwein C, Rakemann T, Malek NP, Plumpe J, Tiegs G, Manns MP: Concanavalin A-induced liver injury triggers hepatocyte proliferation. J Clin Invest 1998, 101:1960-1969

13. Kunstle G, Hentze H, Germann PG, Tiegs G, Meergans T, Wendel A: Concanavalin A hepatotoxicity in mice: tumor necrosis factor-mediated organ failure independent of caspase-3-like protease activation. Hepatology 1999, 30:1241-1251

14. Zheng SJ, Wang P, Tsabary G, Chen YH: Critical roles of TRAIL in hepatic cell death and hepatic inflammation. J Clin Invest 2004, 113:58-64

15. Maeda S, Chang L, Li ZW, Luo JL, Leffert H, Karin M: IKKbeta is required for prevention of apoptosis mediated by cell-bound but not by circulating TNFalpha. Immunity 2003, 19:725-737

16. Kucharczak J, Simmons MJ, Fan Y, Gelinas C: To be, or not to be: NF-kappaB is the answer-role of Rel/NF-kappaB in the regulation of apoptosis. Oncogene 2003, 22:8961-8982

17. Liu H, Lo CR, Czaja MJ: NF-kappaB inhibition sensitizes hepatocytes to TNF-induced apoptosis through a sustained activation of JNK and C-Jun. Hepatology 2002, 35:772-778

18. Schwabe RF, Uchinami H, Qian T, Bennett BL, Lemasters JJ, Brenner DA: Differential requirement for C-Jun NH2-terminal kinase in TNFalpha- and Fas-mediated apoptosis in hepatocytes. FASEB J 2004, 18:720-722

19. Wang Y, Singh R, Lefkowitch JH, Rigoli RM, Czaja MJ: Tumor necrosis factor-induced toxic liver injury results from JNK2-dependent activation of caspase-8 and the mitochondrial death pathway. J Biol Chem 2006, 281:15258-15267

20. Schuchmann M, Varfolomeev EE, Hermann F, Rueckert F, Strand D, Koehler H, Strand S, Lohse AW, Wallach D, Galle PR: Dominant negative MORT1/FADD rescues mice from CD95 and TNF-induced liver failure. Hepatology 2003, 37:129-135

21. Wang K, Yin XM, Chao DT, Milliman CL, Korsmeyer SJ: BID: a nove BH3 domain-only death agonist. Genes Dev 1996, 10:2859-2869

22. Chavolla-Calderon M, Bayer MK, Fontan JJ: Bone marrow transplantation reveals an essential synergy between neuronal and hemopoietic cell neurokinin production in pulmonary inflammation. J Clin Invest 2003, 111:973-980 
23. Chen X, Ding WX, Ni HM, Gao W, Shi YH, Gambotto AA, Fan J, Beg AA, Yin XM: Bid-independent mitochondrial activation in tumor necrosis factor alpha-induced apoptosis and liver injury. Mol Cell Biol 2007, 27:541-553

24. Davis RJ: Signal transduction by the JNK group of MAP kinases. Cell 2000, 103:239-252

25. Liu J, Minemoto Y, Lin A: c-Jun N-terminal protein kinase 1 (JNK1), but not JNK2, is essential for tumor necrosis factor alpha-induced c-Jun kinase activation and apoptosis. Mol Cell Biol 2004, 24:10844-10856

26. Gantner F, Leist M, Lohse AW, Germann PG, Tiegs G: Concanavalin A-induced T-cell-mediated hepatic injury in mice: the role of tumor necrosis factor. Hepatology 1995, 21:190-198

27. Hong F, Jaruga B, Kim WH, Radaeva S, El-Assal ON, Tian Z, Nguyen VA, Gao B: Opposing roles of STAT1 and STAT3 in T cell-mediated hepatitis: regulation by SOCS. J Clin Invest 2002, 110:1503-1513

28. Streetz K, Fregien B, Plumpe J, Korber K, Kubicka S, Sass G, Bischoff SC, Manns MP, Tiegs G, Trautwein C: Dissection of the intracellular pathways in hepatocytes suggests a role for Jun kinase and IFN regulatory factor- 1 in Con A-induced liver failure. J Immunol 2001, 167:514-523

29. Lemasters JJ, Nieminen AL, Qian T, Trost LC, Elmore SP, Nishimura Y, Crowe RA, Cascio WE, Bradham CA, Brenner DA, Herman B: The mitochondrial permeability transition in cell death: a common mechanism in necrosis, apoptosis and autophagy. Biochim Biophys Acta 1998, 1366:177-196

30. Theruvath TP, Snoddy MC, Zhong Z, Lemasters JJ: Mitochondrial permeability transition in liver ischemia and reperfusion: role of c-Jun N-terminal kinase 2. Transplantation 2008, 85:1500-1504

31. Tournier C, Hess P, Yang DD, Xu J, Turner TK, Nimnual A, Bar-Sagi D, Jones SN, Flavell RA, Davis RJ: Requirement of JNK for stressinduced activation of the cytochrome c-mediated death pathway. Science 2000, 288:870-874

32. Irmler M, Thome M, Hahne M, Schneider P, Hofmann K, Steiner V, Bodmer JL, Schroter M, Burns K, Mattmann C, Rimoldi D, French LE,
Tschopp J: Inhibition of death receptor signals by cellular FLIP Nature 1997, 388:190-195

33. Ding WX, Ni HM, DiFrancesca D, Stolz DB, Yin XM: Bid-dependent generation of oxygen radicals promotes death receptor activationinduced apoptosis in murine hepatocytes. Hepatology 2004, 40:403-413

34. Chang L, Kamata H, Solinas G, Luo JL, Maeda S, Venuprasad K, Liu YC, Karin M: The E3 ubiquitin ligase itch couples JNK activation to TNFalpha-induced cell death by inducing c-FLIP(L) turnover. Cell 2006, 124:601-613

35. Sabapathy K, Hochedlinger K, Nam SY, Bauer A, Karin M, Wagner EF: Distinct roles for JNK1 and JNK2 in regulating JNK activity and c-Jun-dependent cell proliferation. Mol Cell 2004, 15:713-725

36. Tsuruta F, Sunayama J, Mori Y, Hattori S, Shimizu S, Tsujimoto Y, Yoshioka K, Masuyama N, Gotoh Y: JNK promotes Bax translocation to mitochondria through phosphorylation of 14-3-3 proteins. EMBO J 2004, 23:1889-1899

37. Gunawan BK, Liu ZX, Han D, Hanawa N, Gaarde WA, Kaplowitz N c-Jun N-terminal kinase plays a major role in murine acetaminophen hepatotoxicity. Gastroenterology 2006, 131:165-178

38. De Smaele E, Zazzeroni F, Papa S, Nguyen DU, Jin R, Jones J, Cong R, Franzoso G: Induction of gadd45beta by NF-kappaB down-regulates pro-apoptotic JNK signalling. Nature 2001, 414:308-313

39. Tang G, Minemoto $Y$, Dibling B, Purcell NH, Li Z, Karin M, Lin A Inhibition of JNK activation through NF-kappaB target genes. Nature 2001, 414:313-317

40. Kamata H, Honda S, Maeda S, Chang L, Hirata H, Karin M: Reactive oxygen species promote TNFalpha-induced death and sustained JNK activation by inhibiting MAP kinase phosphatases. Cell 2005, 120:649-661

41. Pham CG, Bubici C, Zazzeroni F, Papa S, Jones J, Alvarez K Jayawardena S, De Smaele E, Cong R, Beaumont C, Torti FM, Torti SV, Franzoso G: Ferritin heavy chain up-regulation by NF-kappaB inhibits TNFalpha-induced apoptosis by suppressing reactive oxygen species. Cell 2004, 119:529-542 